\title{
CAMR: Congestion-Aware Multi-Path Routing Protocol for Wireless Mesh Networks
}

\author{
Seowoo Jang*, Seok-Gu Kang** and Sung-Guk Yoon ${ }^{\dagger}$
}

\begin{abstract}
The Wireless Mesh Network (WMN) is a multi-hop wireless network consisting of mesh routers and clients, where the mesh routers have minimal mobility and form the backbone. The WMN is primarily designed to access outer network to mesh clients through backhaul gateways. As traffic converges on the gateways, traffic hotspots are likely to form in the neighborhood of the gateways. In this paper, we propose Congestion Aware Multi-path Routing (CAMR) protocol to tackle this problem. Upon congestion, CAMR divides the clients under a mesh STA into two groups and returns a different path for each group. The CAMR protocol triggers multi-path routing in such a manner that the packet reordering problem is avoided. Through simulations, we show that CAMR improves the performance of the WMN in terms of throughput, delay and packet drop ratio.
\end{abstract}

Keywords: Congestion control, Multi-path routing, Wireless mesh network

\section{Introduction}

Wireless mesh networks (WMNs) are one of promising communication technologies for smart grid neighborhood area network (NAN) [1] since it is a cost effective solution with dynamic self-organization, self-configuration, and high scalability services [2]. A WMN is comprised of a root mesh station (STA), mesh STAs, and clients as shown in Fig. 1. In this example, mesh STAs and clients are smart meters and smart appliances, respectively [3]. Root mesh STAs and mesh STAs are stationary and form the wireless backbone. Any mesh STA provides the access point for clients and the root mesh STA is a gateway which is connected to outside the network, i.e., wide area network (WAN), and plays the role of the backhaul gateway.

To select a path from a source to a destination, IEEE 802.11s based WMNs use hybrid wireless mesh protocol (HWMP) as the default routing protocol [4]. Since mesh STAs are traditional layer 2 devices, Medium Access Control (MAC) layer path selection protocol is used in the IEEE 802.11s standard. Also, MAC addresses are used in routing process. HWMP combines the reactive and proactive routings. For the reactive and proactive protocols, it uses Ad-hoc On-demand Distance Vector (AODV) [5] type routing and tree based routing, respectively.

Numerous channel assignment and routing schemes have been proposed for WMNs in order to achieve high capacity. The proliferation of channel assignment approaches largely stems from the possibility of assigning orthogonal channels to different network interface cards

\footnotetext{
$\dagger$ Corresponding Author: Dept. of Electrical Engineering, Soongsil University, Korea. (sgyoon@ssu.ac.kr)

* Samsung Electronics, Korea. (swjang@netlab.snu.ac.kr)

** Dept. of Electrical Engineering, Soongsil University, Korea. (vtz@naver.com)

Received: November 16, 2015; Accepted: August 10, 2016
}

(NICs). Also, numerous routing schemes have been proposed to solve the congestion problem in WMNs $[6,7-$ 9]. This is because the root mesh STAs typically become the most congested part in WMNs, since most traffic goes to the root mesh STAs. This type of congestion degrades the overall performance in WMNs. However, even with multiple root mesh STAs, the problem cannot be perfectly solved $[10,11]$.

Moreover, choosing a best path between each pair of mesh STA and the root mesh STA in the routing protocol can render some intermediate mesh STAs to be overloaded while letting others remain idle. The uneven distribution of the traffic load can be addressed by routing. In addition, multi-path routing in WMN can be used to improve the network utilization by distributing traffics into multiple paths $[12,13]$.

Although a multi-path routing scheme can alleviate congestion, existing approaches suffer from the packet reordering problem, that is, each packet can traverse a different path. For example, suppose there are two distinct paths (path 1 and 2 in Fig. 1) from a source to a destination

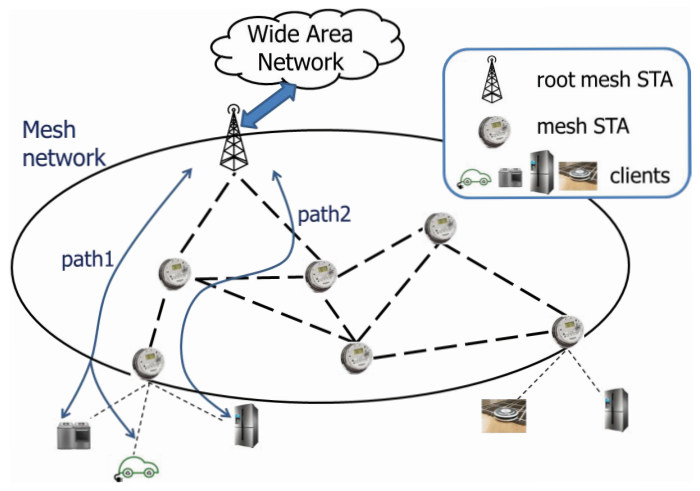

Fig. 1. Example of a wireless mesh network in smart grid 
and path 2 have larger delay. If the source node alternates the routing choice between the two paths for three packets (paths of 1, 2, and 1 for the first, second, third packets, respectively), the second packet might arrive later than the third.

Thus, in this paper, we propose the Congestion Aware Multi-path Routing (CAMR) protocol to resolve congestion in WMNs while avoiding the packet reordering problem. The CAMR creates alternative routing path when congestion is detected while previous multi-path routings do not consider congestion. The novelty of the proposal is the clever use of the Locally Administered Address (LAA) [14] in multi-path routing. It facilitates the classification of packets into corresponding flows ${ }^{1}$ and ensures that all packets in the same flow are forwarded along the same path. As well, the grouping leads to the reduction of the routing table size, improving the scalability. In this paper, we assume single root mesh STA, but this approach can be easily extended to a scenario with multiple root mesh STAs or with multiple orthogonal channels.

The rest of this paper is organized as follows. In Section 2 , related work of the paper is summarized. Then, we detail the CAMR protocol and its operation in Section 3. In Section 4, we evaluate our protocol and present simulation results. Finally, Section 5 concludes the paper.

\section{Related Work}

Recently, researches on WMNs with smart grid have been addressed to support the smart grid specific communication requirements, such as reliability [15] or security issue [16]. Also, load balancing in communication network within smart grid has attracted great deal of interests [17].

Load balancing is one of the core technologies for WMN because traffics in WMN tend to be concentrated to some important links near the root mesh STA. Research on the load balancing can be classified into three categories: gateway-based, mesh-router-based, and path-based load balancing schemes [18]. In the gateway-based load balancing scheme, there are more than one root mesh STAs in WMN, and each client chooses a less congested root mesh STA $[10,11]$. Next load balancing scheme, which is rather link-centric, is the mesh-router-based scheme in which each mesh router decides next router to avoid congestion [17, 19]. Finally, in the path-based load balancing scheme, each client has its own IP address, so the network chooses the best end-to-end route for each client [20].

Another classification of previous routing work is based on whether the routing is performed on L2 (MAC layer) or

\footnotetext{
1 The definition of "flow" is inherently elastic. In this paper we use the term to mean the aggregated traffic that follows the same path from a mesh STA to the root mesh STA. We use it interchangeably with the term "group" throughout the paper.
}

L3 (network layer). Generally, to adopt the path-based load balancing, the WMN supports L3 routing. On the other hand, mesh-router-based load balancing scheme is used with L2 routing. Although L3 routing is more flexible for multi-path routing [20], L2 routing is simpler and the IEEE 802.11 s based WMN operates on top of L2 routing [4]. A survey work of WMN testbed has shown that some WMNs only support L2 routing [21]. Therefore, L2 load balancing routing using multi-path is important.

The proposed CAMR can be classified as a multi-path routing and mesh-router-based load balancing using L2 routing. It tackles packet reordering problem by allocating different routing path for each group. Therefore, in order delivery within each group is guaranteed. In the following sections, we detail the CAMR more specifically.

\section{CAMR: Congestion Aware Multi-path Routing}

In this section, we present the CAMR protocol, which is based on AODV. The proposed CAMR protocol, however, is different in terms of the routing metric and how the routing table entries are managed for multi-path routing.

\subsection{Group policy}

The CAMR group policy is how the mesh STA manages the connected clients. All clients connected to the same mesh STA are collectively called a group. For each group, a group address which is a Locally Administered Address (LAA) is assigned [14]. The LAA is a private MAC address. When a mesh STA $i$ joins the WMN, it obtains a group address $G_{m}(i)$ on behalf of its clients from the root mesh STA, which also creates a corresponding one $G_{\text {root }}(i)$ for itself. Notice that the root mesh STA is represented by multiple group addresses, and there is oneto-one mapping between a mesh STA group address and the corresponding root mesh STA group address: $G_{m}(i) \Leftrightarrow G_{\text {root }}(i), \forall i \in N$, where $N$ denotes the set of group addresses for mesh STAs. Then the root mesh STA can communicate with the mesh STA (in fact, its clients through the mesh STA) using $G_{m}(i)$, and the mesh STA does with the root mesh STA $i$ using $G_{\text {root }}(i)$. The clients connected to the mesh STA $i$ need not know $G_{\text {root }}(i)$ to communicate to the outer network.

As we will show later, the mesh STA intelligently performs any necessary translation, providing transparency to the clients. Client devices, therefore, do not need to be aware of the CAMR protocol, and can simply 'plug and play'. In the downstream direction, the root mesh STA uses $G_{m}(i)$ to reach the clients under the mesh STA $i$. We will discuss the establishment and the use of the mapping in more detail below. Importantly note that a use of the group address leads to the reduction of the L2 routing table size, as the routing table is populated with only group addresses. 


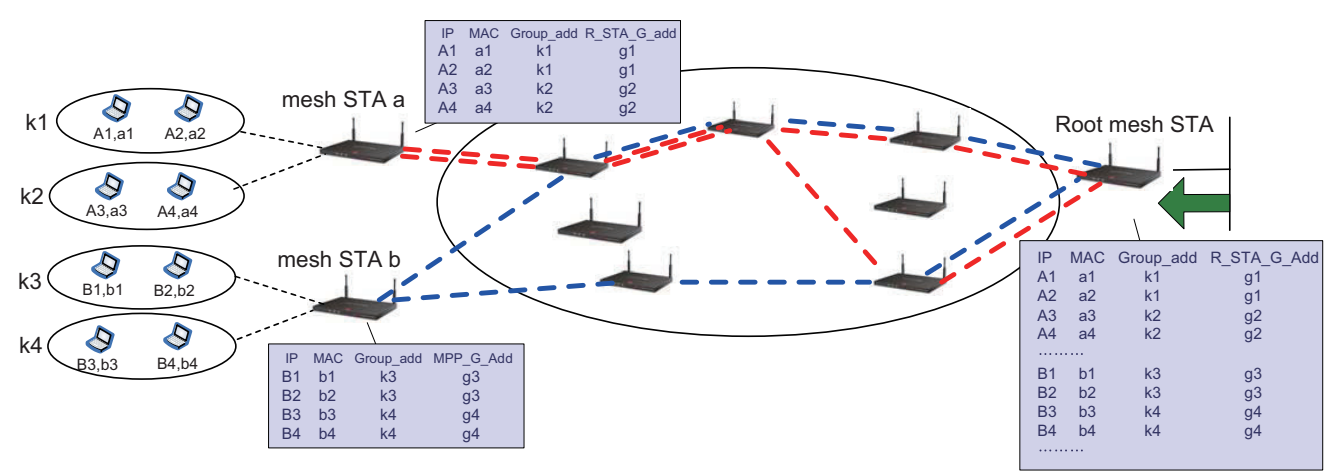

Fig. 2. Operation of root mesh STA and mesh STA with CAMR. The routing tables consist of group address

\subsection{The roles of root mesh STA and mesh STA in group-based routing}

After obtaining a group address, the mesh STA finds the routing path towards the root mesh STA. This part of the CAMR protocol is identical to AODV. For this, the mesh STA transmits an RREQ towards the root mesh STA. After receiving the Route Request (RREQ), the root mesh STA responds with a Route Reply (RREP) towards the requesting mesh STA. Note that in CAMR, multiple paths are created on demand only when congestion is occurred. Thus, initially, it starts with a single path between the newly joining mesh STA and the root mesh STA.

When a mesh STA relays/receives the RREP, the routing table entry for the route is created. The routing table consists of two fields: the destination address and the next hop. For downstream (root mesh STA $\rightsquigarrow$ mesh STA), the destination address field in the routing table is set to the group address of the mesh STA, i.e., $G_{m}(i)$. At the same time, for upstream (mesh STA $\rightsquigarrow$ root mesh STA), the destination is set to the group address of the root mesh STA, i.e., $G_{\text {root }}(i)$. Due to this group-specific, co-located, and bi-directional mapping, the downstream and upstream routing paths concur for the given group. In addition to the group address $G_{m}(i)$, the root mesh STA can assign extra group addresses to the mesh STAs later for multipath routing.

Although group addresses are used in routing, the root mesh STA should have additional information on each client $c$ for address resolution in the routing table. Suppose a client $c$ associates with a mesh STA $i$. The root mesh STA should have the IP address of the client, i.e., $I(c)$, the corresponding mesh STA group address $G_{m}(i)$, and the root mesh STA group address corresponding to the mesh STA group address $G_{\text {root }}(i)$. Using this address resolution information, the mesh STA can translate the IP address of the client to the routable group address. Namely, when the root mesh STA receives packets from outside of the mesh network destined to the client with IP address $I(c)$, the following translation is performed ${ }^{2}: I(c) \Rightarrow G_{m}(i)$.

${ }^{2}$ In this sense, the WMN that uses L2 routing as a whole is a single IP subnet.

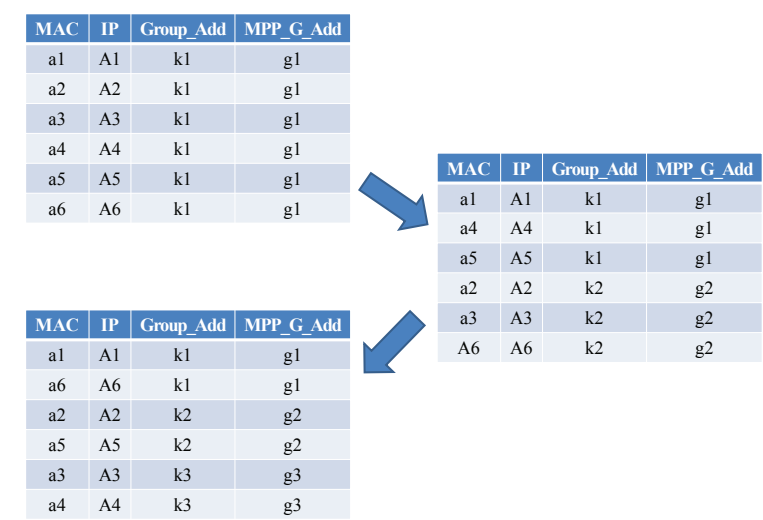

Fig. 3. The mesh STA routing table upon group spliting

Fig. 2 illustrates the roles of mesh STA and root mesh STA in CAMR. In the example, the clients are divided into two groups according to the mesh STA association and grouping policy. The mesh STA $a$ has group addresses $k 1$ and $k 2$, while $b$ has $k 3$ and $k 4$. When the root mesh STA receives a packet destined to $B 3$ from outside of the mesh network, it finds $k 4$ in the table and sets the destination MAC address of the packet to $k 4$. When the mesh STA $b$ receives the packet, it looks up its routing table and replaces $k 4$ with the MAC address of $B 3$, i.e., $b 3$ so that the packet is correctly delivered to $B 3$.

On the other hand, in the case of upstream transmission from $B 3$ to the outer network, the mesh STA should know that the packet is sent to the root mesh STA first. Then, it also checks the source IP address of the sender, i.e., $B 3$ so that the MAC address of the root mesh STA is set to $g 4$. This group address will correctly route the packet to the root mesh STA.

The mesh STA has to manage its clients and maintain the pertinent client information in the routing table for correct routing and load balancing. First, it has to keep the root mesh STA group address which was obtained when the mesh STA joined the network. In addition, it should keep the additional group addresses obtained on congestion episodes for multi-path routing as well. Second, it should know the IP and MAC addresses of the clients, and the pair of group address-group address for the routing between the root mesh STA and the clients. Third, the mesh STA should 


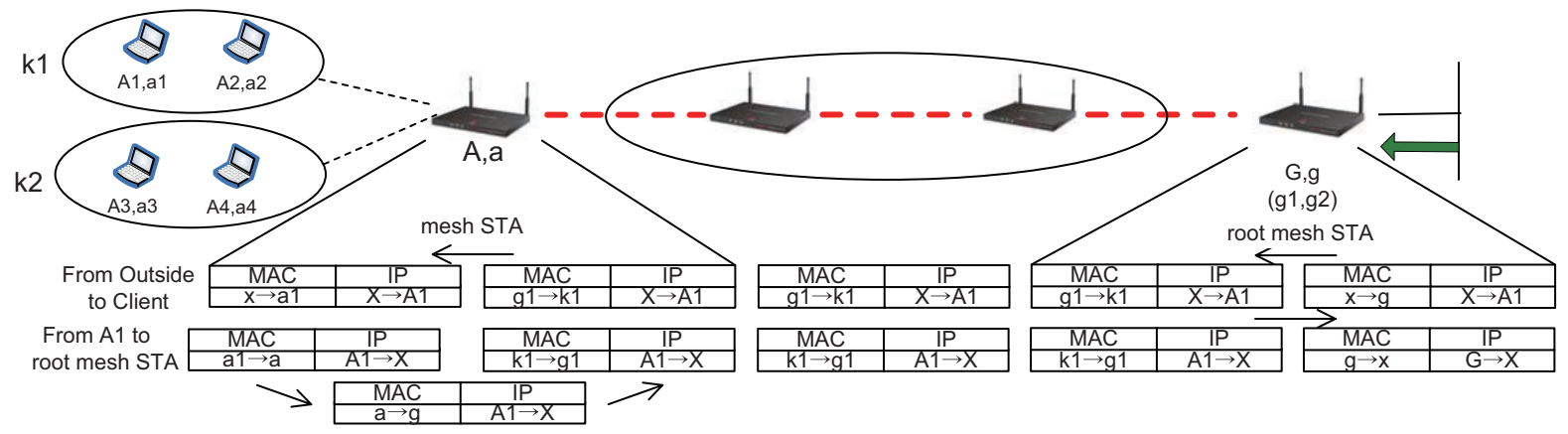

Fig. 4. Operation of root mesh STA and mesh STA with CAMR

record the traffic rate per group address. When congestion occurs and the MAC receives an additional group address from the root mesh STA for multi-path routing, the mesh STA (further) splits the clients into more groups in such a manner that the traffic rate of the groups is similarly matched.

Fig. 3 shows a simple example of group splitting as seen by the mesh STA routing table. Here, the original group mapping of the clients is $a 1, a 2, \cdots, a 6 \Rightarrow k 1$ (upper left). After obtaining another group address, $k 2$, the clients are split into two groups. Now, we have $a 1, a 4, a 5 \Rightarrow k 1$ and $a 2, a 3, a 6 \Rightarrow k 2$ (right), so that the aggregate traffic rate of the groups is maintained at a comparable level. Also, the group address mapping is correspondingly split. Getting more group addresses similarly leads to the traffic-based splitting of existing groups and membership readjustment (lower left).

Fig. 4 shows a detailed example of packet routing in the network of Fig. 2. Here, we assume that the host in the outer network that corresponds with client $A 1$ (IP address is $X$ ). In the figure, the notation $\alpha \rightarrow \beta$ represents the fact that $\alpha$ is the source address, and $\beta$ is the destination address in the packet header, both for the IP header and the MAC header. For the MAC layer, source addresses are mostly irrelevant, in which case we simply refer to it as $x$ although it keeps changing as links are crossed.

When the root mesh STA receives an incoming packet destined to $A 1$, it finds the mapping $A 1(a 1) \Rightarrow k 1$. Then it changes the destination MAC address of received packet to $k 1$. The packet is routed to the destination mesh STA $A$. The mesh STA recovers the original MAC address for $A 1$ from the routing table mapping $A 1 \Rightarrow a 1$, which is overwritten on the destination MAC field of the packet, and delivered. Conversely, the packet from $A 1$ to the root mesh STA (and onward to the Internet) is given $g 1$ as the destination MAC address according to the routing table at mesh STA $A$.

\subsection{CAMR protocol operation for multi-path routing}

We consider how the routing framework is extended to accommodate the multi-path routing and load balancing, while retaining the design objective of ordered packet delivery through group-based routing.

\subsubsection{Congestion detection}

CAMR addresses the congestion problem and attempts to increase the performance of the network when congestion occurs within the network. Specifically, each mesh STA detects congestion by observing the queue occupation of its interface.

In CAMR, congestion is detected when the queue length of an interface is above a certain threshold. To avoid an unstable effect due to a momentary change in the queue length, we use an exponential moving average method for smoothing; in other words, the queue length used in this consideration is given by:

$$
q^{*}(t)=\alpha q(t)+(1-\alpha) q^{*}(t-1), \quad(0<\alpha<1)
$$

where $q(t)$ is the measured queue length while $q^{*}(t)$ is the smoothed queue length at time $t . \alpha$ is the moving average weight. When $q^{*}(t)$ is larger than a predetermined threshold, congestion is detected.

\subsubsection{Multi-path creation}

If the congestion is detected by a intermediate mesh STA, it checks which group address has the most traffic, and informs the mesh STA associated with the address. Then the notified mesh STA should split the group in question according to the procedure illustrated in Fig. 3. In case of the mesh STA with client(s) detected congestion, it immediately initiates the splitting process.

Fig. 5 illustrates the process. In Fig. 5(a), mesh STA $X$ is transmitting packets to root mesh STA $Y$ through routing path (1), that is, $X \rightarrow D \rightarrow B \rightarrow Y$. Mesh STA $A$ is also transmitting to mesh STA $Y$, whose path is partially overlapped with (1), namely $A \rightarrow B \rightarrow Y$. Suppose $B$ experiences congestion on the link between $B$ and $Y$, and the traffic rate from $X$ is larger than that from $A$. Then $B$ informs $X$ by transmitting Congestion_notify message as 


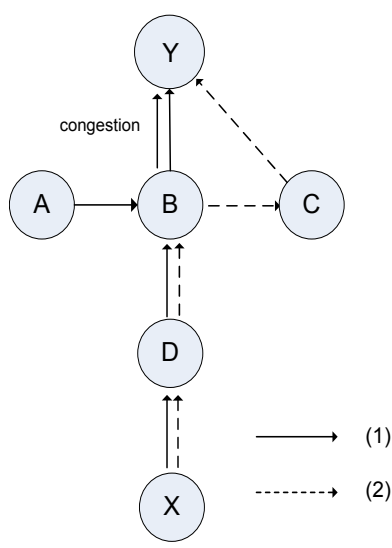

(a) Alternate path setup

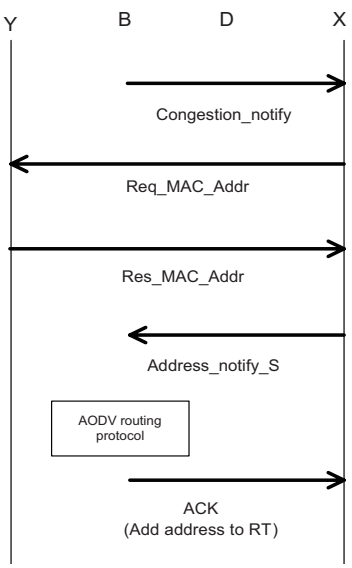

(b) Control message exchange

Fig. 5. Routing and control message exchange for alternate path creation

shown in Fig. 5(b). The Congestion_notify message carries $B$ 's address. When $X$ receives Congestion_notify message, it transmits in response Req_MAC_Addr to the root mesh STA to request another group address. The root mesh STA responds with Res_MAC_Addr, which carries the new group address. $X$ saves the new group address and transmits towards $B$ the Address_notify message that carries the newly created group address.

Upon receiving this information, $B$ finds another routing path to the root mesh STA for the newly split group under $X$ by executing the AODV routing protocol using the new group address. Specifically, $B$ transmits RREQ through each interface except the interface where congestion occurred. Upon finding a new path (2), $B \rightarrow C \rightarrow Y$, it transmits an ACK message to $X$. Then $X$ executes the group policy to subdivide the clients. Finally, packets will be routed multi-path, namely $X \rightarrow D \rightarrow B \rightarrow Y$ (path (1)) and $X \rightarrow D \rightarrow B \rightarrow C \rightarrow Y$ (path (2)). This helps resolve the congestion at $B$.

It is possible that the congested mesh STA will not find an additional alternate path. In such a case, the congested router first attempts to create one at 1-hop upstream on the routing path of the most congested group. For instance, in Fig. 6, $B$ finds that it cannot create an alternate path. Then it transmits a Congestion_notify_B message to the 1-hop upstream $D$ for the most congested group as shown in Fig. 6(a). Then $D$ attempts to perform the multi-path creation process instead of $B$. In this example, $D$ finds a new routing path, $X \rightarrow D \rightarrow C \rightarrow Y$, which $X$ will use in addition to the existing path to transmit packets to the root mesh STA.

If no additional alternate path can be created between the root mesh STA and the mesh STA, the Congestion_notify message ends up being sent to $X$, at which point $X$ terminates the alternate path creation process since no path could be created between the congested mesh STA $(B)$ and the congested mesh STA $(X)$. The congested mesh STA

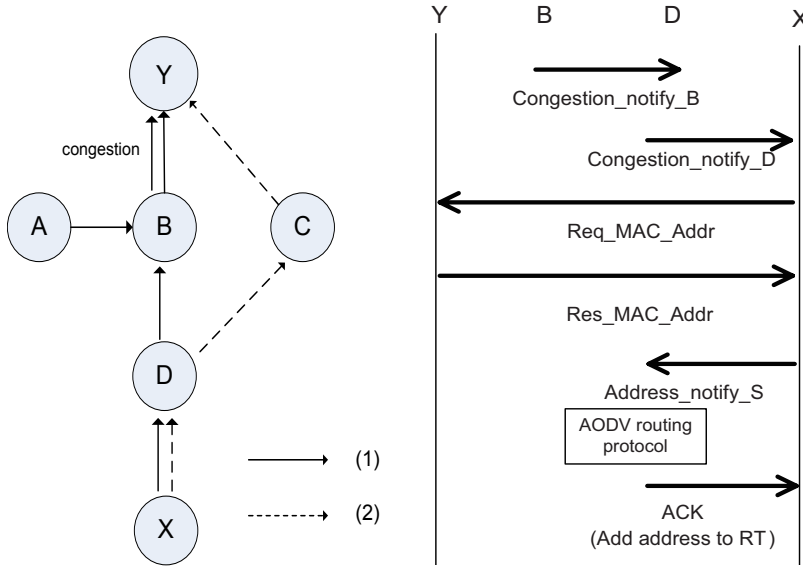

(a) Routing path setup (b) Control message exchange

Fig. 6. Routing and control message exchange in case $B$ cannot create an alternate path

runs a timer, waiting to get Address notify message from the congested mesh STA. If the timer expires while congestion persists, it can re-initiate the whole process with the next most congested group.

\subsubsection{Path shutdown and group merging}

After creating multiple paths, the mesh STA can observe that the traffic from its client(s) decreases. In this case, some multi-paths may have been dismantled, as the alternate paths generally have worse characteristics and consume more network resources (i.e., a larger weighted hop distance or cost). Note that only some mesh STAs that have client(s) can initiate path shutdown or group merging.

For this purpose, we define a threshold value $\theta_{m}$ and the traffic load at the mesh STA $L$. If $L>\theta_{m}$, we keep the current routing paths lest the decrease in the number of paths should cause congestion in the surviving paths. On the other hand, if $L<\theta_{l}$, the mesh STA prefers to use a single path because the amount of traffic is small. The mesh STA dismantles all paths except the least cost path. For this purpose, we let each mesh STA save the minimum cost path to the root mesh STA after finding it initially through AODV.

Finally, if $\theta_{l}<L<\theta_{m}$, some paths need to be dismantled. The determination of the number of paths and the selection of the paths to be purged proceed as follows. We assume that mesh STAs that have client(s) can probe the queue length of each interface of a mesh STA on the current routing paths for their client(s) in a piggybacking manner.

(1) Find the largest smoothed queue length $q_{r}^{*}$ on each currently used routing path $r$.

$$
q_{r}^{*}=\max _{1 \leq h \leq\left|H_{r}\right|}\left\{q_{h, r}^{*}\right\}, \quad r \in H
$$

where $q_{h, r}^{*}$ and $\underline{H}$ are the smoothed queue length of $h$ th 
mesh STA of path $r$ and the set of the multi-paths, respectively, and $\left|H_{r}\right|$ is the hop count of path $r$.

(2) If there is a routing path $r$ such that $q_{r}^{*}=0$, the mesh STA selects the group address whose transmission rate is the lowest. The mesh STA merges this group into the group using the minimum hop path. This step is repeated until $q_{r}^{*} \neq 0$ for $\forall r \in H$.

\section{Performance Evaluation}

In this section, we investigate the performance improvement of the CAMR protocol in terms of networkwide throughput, delay, and packet drop ratio through simulations. The network-wide throughput is defined as the total amount of bytes received at the root mesh STA over the entire simulation duration. And the delay is defined as the time for a packet staying in the WMN, i.e., the time period of a packet experienced from the departure from its associated mesh STA to the arrival to the root mesh STA. Note that the delay of dropped packets are not counted. We compare the proposed CAMR with two single path routing schemes. One is minimum cost path routing which only considers the sum of link status to the destination [9]. The other routing scheme is load balancing routing scheme designed for AMI network [17].

\subsection{Simulation settings}

We implement the CAMR protocol simulator using ns-3. Each node is equipped with three or five radio interfaces and each link is assigned a unique channel to minimize the effect of inter-channel interference [22].

The CAMR protocol is evaluated by two topologies: triangle lattice and random topologies. For the triangle lattice topology, we consider 18 mesh STAs and one root mesh STA as shown in Fig. 7. The bottom six mesh STAs has clients and there are six clients per the bottom mesh STA. The other 11 mesh STAs as relay nodes between

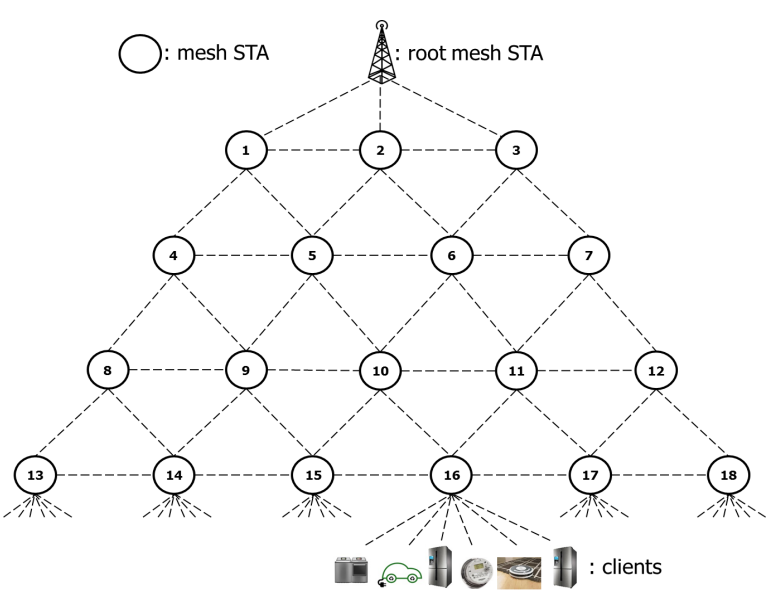

Fig. 7. Triangle lattice topology. Mesh STAs (13-18) are serving six clients clients and the root mesh STA, and the only one root mesh STA acts as a gateway to the outer network. The six clients in mesh STA 16 vary their traffic intensity, while the other clients generate traffic with fixed rate of $200 \mathrm{kbps}$. Transmission distance is set to $110 \mathrm{~m}$, and mesh STAs are placed to have connections only to neighboring nodes. Connections are represented solid line in Fig. 7.

In the random topology, there exist one root mesh STA and 24 mesh STAs in $500 \mathrm{~m} \times 500 \mathrm{~m}$ area. The root mesh STA is located at the center of the area $(250 \mathrm{~m}, 250 \mathrm{~m})$ and mesh STAs are randomly deployed with uniform distribution. The minimum distance of each pair of mesh STAs and the maximum transmission range are set to 100 $\mathrm{m}$ and $150 \mathrm{~m}$, respectively. The bottom mesh STAs have six clients and each client generates $200 \mathrm{kbps}$ traffic as before. But the six clients associated to a particular mesh STA change their traffic intensity.

The transmission capacity of links is set to $11 \mathrm{Mbps}$. The traffic from the clients is UDP/CBR where the packet size is $1 \mathrm{kB}$. And the buffer size at each node is set to 200 packets.

After experimenting with several different values of the moving average weight $\alpha$ (see Eq. (1)), we have set it to 0.5 , which provides a stable smoothed queue length from one instant to another. We assume that the congestion occurs when the smoothed queue length reaches $90 \%$ of the node buffer and that the two threshold values for merging paths, i.e., $\theta_{m}$ and $\theta_{l}$ are set to 0.3 and 0.7 , respectively. The values were found from simulations.

\subsection{Performance results}

Fig. 8 shows the throughput, delay, packet drop ratio for the triangular lattice topology. We plot the performances of the minimum cost path (MCP) routing [9] and the load balancing routing (LBR) [17] with and without CAMR. The traffic load for clients in mesh STA 16 is varied from $200 \mathrm{kbps}$ to $2.2 \mathrm{Mbps}$.

Fig. 8(a) shows the network-wide throughput. As the traffic intensity increases, the throughput performance of MCP is saturated first, and then that of LBR saturates. We observe that the addition of CAMR significantly improves throughput performance. It is because the two single path routings (MCP and LBR) only use one route while CAMR finds another route when the current route is congested. The throughput performance improvements due to addition of CAMR are $48.4 \%$ and $28.2 \%$ for MCP and LBR, respectively.

Fig. 8(b) shows the average delay performance. For all four routing schemes, the delay performances remain very small, i.e., $8 \mathrm{msec}$, when there are no packet drops. When packet droppings exist, delays increase impulsively. In the two single path routings only one step of the delay jump exists because there is just one bottleneck link. However, the average delay with the CAMR scheme shows multiple jump steps because CAMR has different queue states for 
multiple paths. Note that the average delays of single path routings show better performance than those with CAMR when the traffic intensity is higher than $1.8 \mathrm{Mbps}$. This is because the delays of dropped packets are not counted in the average delay.

Fig. 8(c) shows packet drop ratio. It shows the same results to the throughput performance. That is, LBR with CAMR and MCP show the best and the worst performances, respectively. It is because CAMR better utilizes network resources.

We also simulate the case of having three radio interfaces. In the triangular lattice topology, there is almost

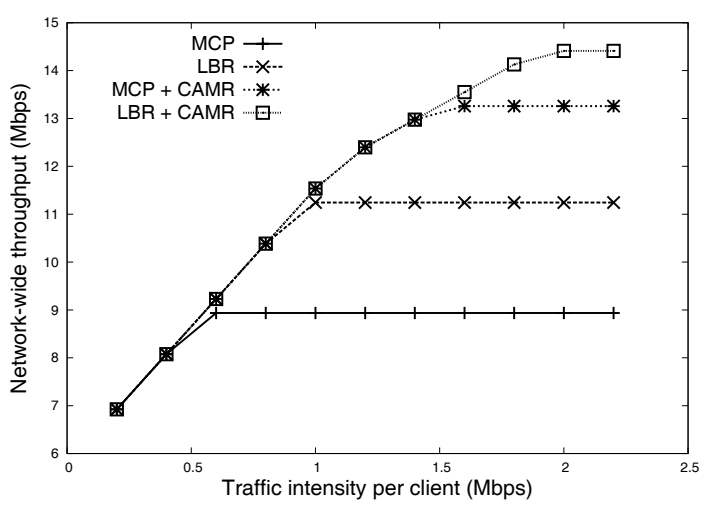

(a) Network-wide throughput

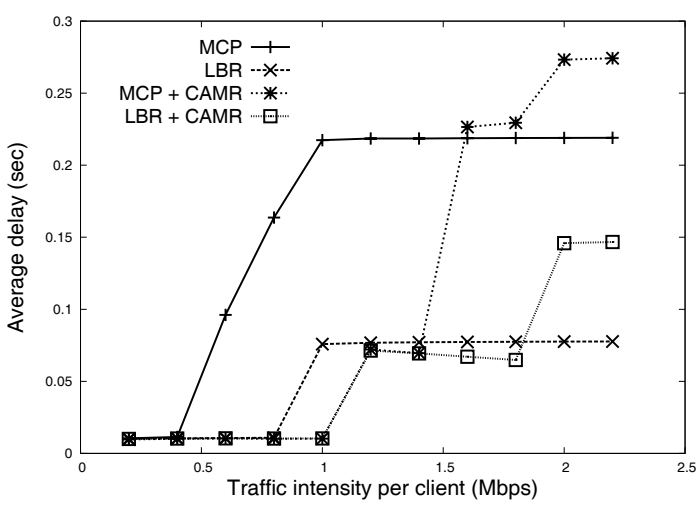

(b) Average delay

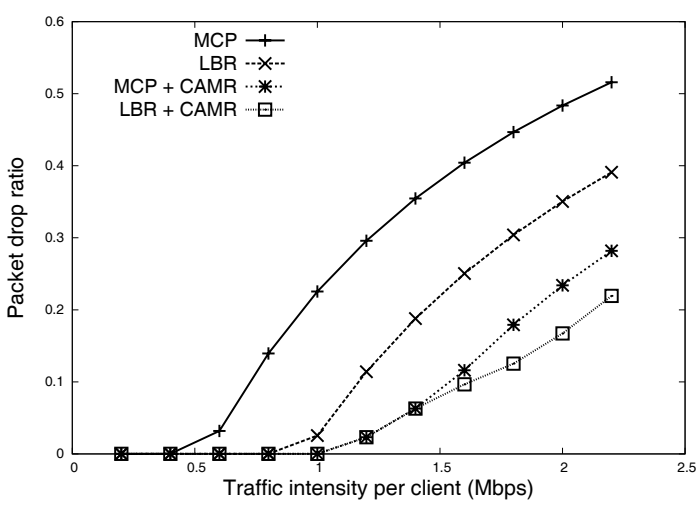

(c) Packet drop ratio

Fig. 8. Performances in the triangular lattice topology no performance degradation by limiting the number of interfaces to three because its simple topological structure allows three independent channels to be enough to utilize alternate route.

Fig. 9 shows the simulation results for the random topology with five radio interfaces. The general tendency of the performances is similar with that of the lattice topology. As shown in Fig. 9(a), the throughput performance improving gap by adding CAMR is better than the lattice topology case. It is because some mesh STAs in the random structure have many routes to the root

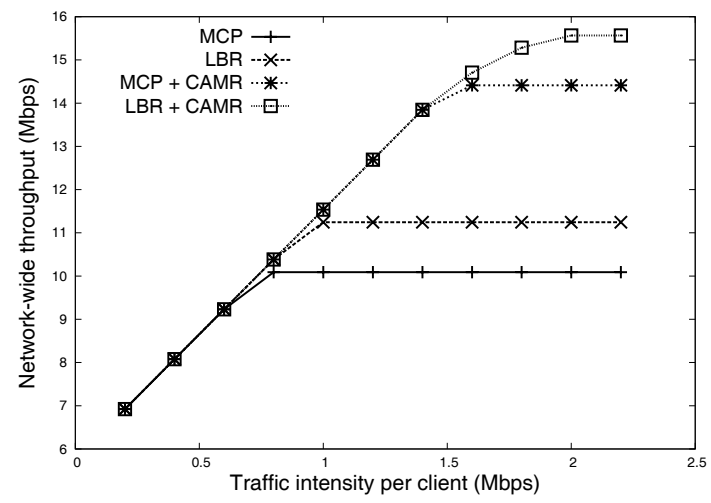

(a) Network-wide throughput

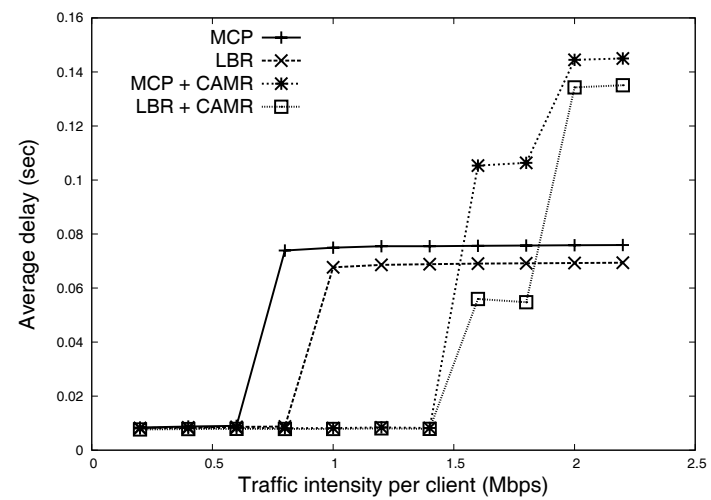

(b) Average delay

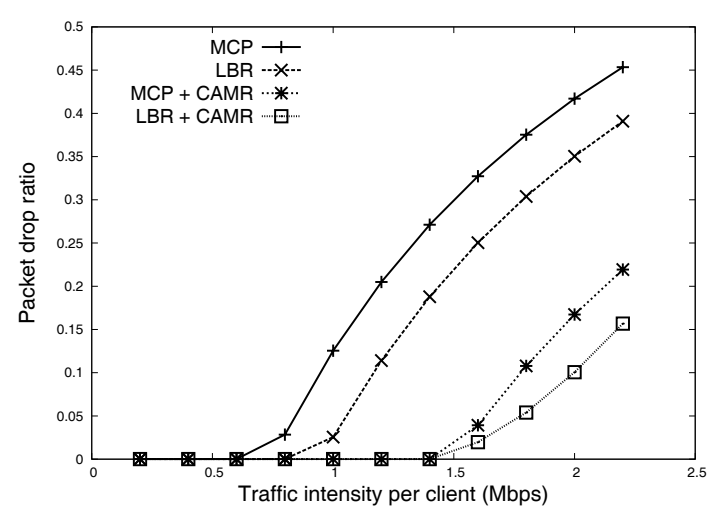

(c) Packet drop ratio

Fig. 9. Performances in the random topology with five radio interfaces 
mesh STA, so the network resource is further utilized through multi-path routing scheme.

In the random topology, we simulate three radio interfaces case too. Because of its similar tendency, we omit the performance graphs. The throughput performance and packet drop ratio decreases and increases, respectively. The maximum throughput performances for MCP, LBR, MCP with CAMR, and LBR with CAMR are $8.94 \mathrm{Mbps,}$ 11.2 Mbps, 12.1 Mbps, and 14.4 Mbps, respectively, which is $11.4 \%, 0 \%, 16.0 \%$, and $7.4 \%$ performance degradations, respectively. In the random topology, some mesh STAs have many links and some has only one links. Therefore, with three radio interfaces, the mesh STAs which have many links cannot be fully utilized. In LBR, there is no performance degradation because single path routing with load balancing does not require many independent channels.

\section{Conclusion}

In this paper, we proposed layer 2 multi-path routing protocol, named Congestion-Aware Multi-path Routing (CAMR), which helps the WMN to cope with traffic hotspots. The proposed scheme finds alternative route when current route suffers from congestion. Although CAMR uses multiple paths to the destination in $\mathrm{WMN}$, it prevents packet reordering problem by controlling the traffic distribution on a client group basis. Through simulations, we demonstrated that the CAMR protocol always provides higher throughout and lower packet drop ratio, especially at higher traffic intensity. Also, it is shown that more number of radio interfaces and orthogonal channels are required to better utilize the benefit of multi-path routing.

\section{Acknowledgements}

This work was supported in part by "Human Resources Program in Energy Technology" of the Korea Institute of Energy Technology Evaluation and Planning (KETEP), granted financial resource from the Ministry of Trade, Industry \& Energy, Republic of Korea (No. 20164010201010), and in part by Basic Science Research Program through the National Research Foundation of Korea (NRF) funded by the Ministry of Science, ICT \& Future Planning (NRF2015R1C1A1A02037774).

\section{References}

[1] W. Meng and R. Ma, and H.-H. Chen "Smart Grid Neighborhood Area Networks: A Survey," IEEE Network, vol. 28, no. 1, pp. 24-32, Jan.-Feb. 2014.

[2] D. Benyamina, A. Hafid, and M. Gendreau, "Wireless Mesh Networks Design - A Survey," IEEE
Communications Survey and Tutorials, vol. 14, no. 2, pp. 299-310, second quarter. 2012.

[3] M. Kuzlu, M. Pipattanasomporn, and S. Rahman, "Communication Network Requirements for Major Smart Grid Applications in HAN, NAN and WAN," Computer Networks, vol. 67, no. 4, pp. 74-86, Jul. 2014.

[4] IEEE 802.11-2012 Standard for Information Technology-Telecommunications and information exchange between systems Local and metropolitan area networks-Specific requirements Part 11: Wireless LAN Medium Access Control (MAC) and Physical Layer (PHY) Specifications. Mar. 2012.

[5] S. Das, C. Perkins and E. Royer, "Ad Hoc on Demand Distance Vector (AODV) Routing," IETF RFC3561, Jul. 2003.

[6] S. Lee and M. Gerla, "Split Multipath Routing with Maximally Disjoint Paths in Ad Hoc Networks," in Proc. IEEE ICC, Jun. 2001.

[7] M. Marina and S. Das, "On-demand Multipath Distance Vector Routing in Ad Hoc Networks," in Proc. of IEEE ICNP, Nov. 2001.

[8] A. Bhorkar, T. Javidi, and A. Snoeren, "Achieving Congestion Diversity in Multi-Hop Wireless Mesh Networks," IEEE Transactions on Mobile Computing, vol. 14, no. 3, pp. 473-483, Mar. 2015.

[9] R. Draves, J. Padhye, and B. Zill, "Routing in Multiradio, Multi-hop Wireless Mesh Networks," in Proc. MobiCom, Sep.-Oct. 2004.

[10] Y. Hu, W. He, S. Yanh and Y. Zhou, "Multi-Gateway Multi-Path Routing Protocol for 802.11s WMN," in Proc. IEEE WiMob, Oct. 2010.

[11] P. Lin, K. H. Yeung and K. Y. Wong, "Multiple Path Routing using Tree-Based Multiple Portal Association for Wireless Mesh Networks," in Proc. IEEE ISWPC, Feb. 2011.

[12] K. Ramachandran, I. Sheriff, E. Belding, K. Almeroth, "A Multi-radio 802.11 Mesh Network Architecture," Mobile Networks and Applications, vol. 13, pp. 132146, 2008.

[13] E. Alotaibi, B. Mukherjee "A Survey on Routing Algorithms for Wireless Ad-Hoc and Mesh Networks," Computer Networks, vol. 56, no. 2, pp. 940965, Feb. 2012.

[14] IEEE Standards Association, [Online]. Available: http://standards.ieee.org/regauth/groupmac/tutorial.ht ml Accessed: Nov. 16, 2015.

[15] J. Kim, D. Kim, K.-W. Lim, Y.-B. Ko, and S.-Y. Lee, "Improving the Reliability of IEEE 802.11s Based Wireless Mesh Networks for Smart Grid Systems," Journal of Communications and Networks, vol. 14, no. 6, pp. 629-639, Dec. 2012.

[16] K. Akkaya, and K. Rabieh, M. Mahmoud, and S. Tonyali, "Customized Certificate Revocation Lists for IEEE 802.11s-based Smart Grid AMI Networks," IEEE Transactions on Smart Grid, vol. 6, no. 5, pp. 
2366-2374, Sep. 2015.

[17] A. Robertsingh, D. Devaraj, R. Narmathabanu, "Development and Analysis of Wireless Mesh Networks with Load-balancing for AMI in Smart Grid," in Proc. CoCoNet, India, Dec. 2015.

[18] B. Das and S. Roy, "Load Balancing Techniques for Wireless Mesh Networks: A Survey," in Proc. ISCBI, Aug. 2013.

[19] L. Ma and M. Denko, "A Routing Metric for LoadBalancing in Wireless Mesh Networks," in Proc. AINAW, May 2007.

[20] J. Gálvez, P. Ruiz, "Efficient Rate Allocation, Routing and Channel Assignment in Wireless Mesh Networks Supporting Dynamic Traffic Flows," Ad Hoc Networks, vol. 11, no. 6, pp. 1765-1781, 2013.

[21] B. Blywis, M. Günes, F. Juraschek, J. Schiller, "Trends, Advances, and Challenges in Testbed-based Wireless Mesh Network Research," Mobile Networks and Applications, vol. 10, pp. 315-329, 2010.

[22] H. Skalli, S. Ghosh, S. Das, L. Lenzini, M. Conti, "Channel Assignment Strategies for Multiradio Wireless Mesh Networks: Issues and Solutions," IEEE Communications Magazine, vol. 45, no. 11, pp. 86-95, Nov. 2007.

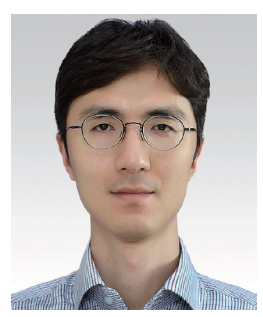

Seowoo Jang He received B.S. and M.S. degrees in the school of Electrical Engineering \& Computer Science, Seoul National University, Korea in 2007 and 2009, respectively. He also received the Ph.D. degree from Seoul National University in 2015. He was also a visiting scholar in University of Michigan, Ann Arbor, in 2014-2015. From 2015, he worked as a post-Doc researcher at Seoul National University. $\mathrm{He}$ is currently a senior software engineer in Samsung Electronics. His research interests include wireless MAC protocol design, resource management, network optimization and machine learning designs in cellular LTE, nextgeneration WLAN systems and IoT systems. His research area also includes vehicular communication system.

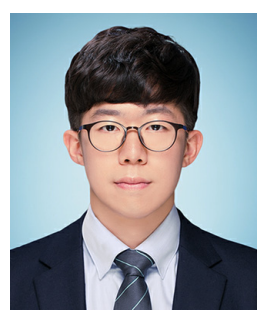

Seok-Gu Kang He received the B.S. degree in electrical engineering from Soongsil University, Korea, in 2015. Currently, he is working toward the M.S. degree in electrical engineering from Soongsil University. His research interests include smart grid, mircrogrid, and load forecasting.

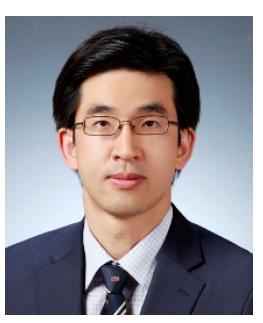

Sung-Guk Yoon He received the B.S. and Ph.D. degrees from Seoul National University, Seoul Korea, in 2006 and 2012, respectively. From 2012 to 2014, he was a Postdoctoral Researcher at the same university. He is currently with Sonngsil University as an Assistant Professor since March 2014. His research interests include smart grid and power line communications. 